\title{
Inovações tecnológicas na cadeia produtiva de feijão
}

\section{Technological innovations in bean processing}

\author{
Revenli Fernanda do Nascimento \\ Universidade Tecnológica Federal do Paraná - UTFPR - Ponta Grossa - Brasil \\ revenli fernanda@hotmail.com \\ Adriane Bonfim Gavron \\ Universidade Tecnológica Federal do Paraná - UTFPR - Ponta Grossa - Brasil \\ adrianebonfim@yahoo.com.br \\ Juliana Vitória Messias Bittencourt \\ Universidade Tecnológica Federal do Paraná - UTFPR - Ponta Grossa - Brasil \\ julianavitoria@utfpr.edu.br
}

\section{Resumo}

Com a grande demanda de mercado para o feijão, as agroindústrias têm investido em inovações tecnológicas, em razão da necessidade de manter-se competitivas no atual cenário socioeconômico, além de atender as mudanças nos gostos dos consumidores e produzir alimentos seguros e de qualidade. Desta forma este trabalho teve por objetivo investigar quais inovações tecnológicas de processos e produtos estão sendo empregadas no setor agroindustrial de processamento de feijão, por meio da avaliação dessas inovações tecnológicas implementadas numa agroindústria desse setor situada no estado do Paraná. $O$ estudo de caso realizado permitiu intuir o elevado grau de investimento em inovações tecnológicas na agroindústria objeto de estudo. As inovações tecnológicas de processos referem-se aos processos de limpeza, secagem, classificação e empacotamento, inteiramente automatizados. As inovações tecnológicas de produtos referem-se ao feijão Premium e o feijão vermelho, atualmente comercializados pela empresa. As inovações tecnológicas implementadas permitiram à empresa minimizar os desperdícios, maximizar o lucro, otimizar a produtividade em grande escala, conquistar novos mercados e melhorar a performance organizacional. Nesse contexto, pode-se concluir que a implementação de inovações no setor produtivo é necessária às empresas que desejam se manter competitivas no mercador consumidor, principalmente para aquelas em um segmento com contínuo crescimento, como o feijão.

Palavras-chave: inovações tecnológicas, processos, produtos, feijão.

\begin{abstract}
With the large market demand for beans, agribusinesses have invested in technological innovations, because of the need to remain competitive in the current socio-economic scenario, as well as meet the changes in consumer tastes and produce safe and quality food. Thus this study aimed to investigate which technological innovation processes and products are being used in the agro-industrial sector of bean processing, through the evaluation of these technological innovations implemented in this sector agribusiness
\end{abstract}


located in the state of Paraná. The conducted case study allowed intuit the high level of investment in technological innovation in agribusiness object of study. The technological process innovations relate to cleaning, drying, sorting and packaging, fully automated. Technological innovations in products refer to beans Premium and red beans, currently marketed by the company. The implemented technological innovations enabled the company to minimize waste, maximize profit, optimize large-scale productivity, reach new markets and improve organizational performance. In this context, it can be concluded that the implementation of innovations in the productive sector is essential for businesses that want to remain competitive in the consumer merchant, especially for those in a segment with continued growth, such as beans.

Keywords: technological innovations, processes, products, bean.

\section{Introdução}

O feijão comum (Phaseolus vulgaris $L$.) é uma leguminosa cultivada há milhares de ano pela espécie humana, sem no entanto, um histórico definido sobre a sua origem e de quando o homem começou a utilizá-lo como cultura doméstica (FACCION, 2011). Sabese atualmente, que o feijão é amplamente consumido em todo o mundo, considerado importante fonte de proteínas, hidratos de carbono, vitaminas, minerais e fibras, constituindo-se um alimento de base primária na dieta, em vários países ocidentais. (KHANALA et al., 2014).

Segundo o Ministério da Agricultura, Pecuária e Abastecimento (MAPA), o Brasil é o maior produtor mundial de feijão com produção média anual de 3,5 milhões de toneladas, sendo os maiores produtores o estado do Paraná, que colheu 298 mil toneladas na safra 2009/2010 e o estado de Minas Gerais, com a produção de 214 mil toneladas no mesmo período. Essa produção abastece apenas o mercado interno, de acordo com a Pesquisa de Orçamento Familiar (POF) do Instituto Brasileiro de Geografia e Estatística (IBGE, 2011) onde o consumo diário per capita para feijão é de 182,9 g/ dia, juntamente com o arroz (160,3 g/ dia), compondo a alimentação básica das populações de baixa renda (CARVALHO et al., 2012).

Com a grande demanda de mercado para o feijão, as agroindústrias têm investido em inovações tecnológicas, em razão da necessidade de manter-se competitivas no atual cenário socioeconômico, além de atender às mudanças nos gostos dos consumidores e produzir alimentos seguros e de qualidade (CÁRCEL et al., 2012). Para Oliveira (2003, p.95), com "o surgimento constante de novas tecnologias torna-se conveniente repensar o produto ou o processo de produção e verificar se as necessidades dos clientes podem ser atendidas de uma forma mais plena ou econômica".

A inovação tecnológica é um processo dinâmico, que requer um esforço combinado das funções organizacionais e recursos limitados (OLIVEIRA; ALVES, 2013). A OECD (1997, p. 23) define inovações tecnológicas como "todas as etapas científicas, tecnológicas, organizacionais, financeiras e comerciais que de fato levam, ou pretendem levar, à implantação de produtos ou processos tecnologicamente novos ou aprimorados". Nesta definição podem ser assinalados dois grupos principais de inovações tecnológicas: inovações de processo e inovações de produto. As inovações de processo referem-se à introdução de novos ou melhorados métodos de produção (SNYDER et al., 2016), com a principal finalidade de permitir que a empresa obtenha aumento de produtividade e redução de custos (SANTINI; FILHO; BÁNKUTI, 2006). As inovações de produto são resultado da combinação de fatores que implicam no desenvolvimento de novos produtos ou aperfeiçoamento dos já existentes (DIACONU, 2011), alterações que de maneira geral objetivam a adequação do portfólio de produtos das empresas às novas necessidades dos consumidores (SANTINI; FILHO; BANKUTI, 2006). De acordo com Diaconu (2011), 
as empresas mais inovadoras, buscam introduzir ambos os tipos de inovações ao mesmo tempo, visando à competitividade dos preços e/ou tecnológica.

No processo de desenvolvimento de inovações, seja de produto ou de processo, o acesso à informação técnica e ao conhecimento é de fundamental importância para implementação destas. A informação técnica na maioria das vezes está disponível para todos os agentes no mercado. No entanto, o conhecimento não se encontra totalmente disponível, devido ao esforço necessário para compreendê-lo, explorá-lo e aplicá-lo. (PATEL; PAVITT apud ARUNDEL et al., 1998; SANTINI; FILHO; BÁNKUTI, 2006).

Dessa forma, levando-se em conta a representatividade do feijão no mercado consumidor e a necessidade da implementação de inovações tecnológicas nesta cadeia produtiva, este trabalho teve por objetivo investigar quais inovações tecnológicas de processos e produtos empregadas neste setor, por meio da avaliação realizada em uma agroindústria de processamento de feijão, situada no estado do Paraná.

\section{Metodologia}

Conforme as classificações da pesquisa propostas por Gil (2008), a presente pesquisa caracteriza-se como estudo de caso, visto que busca aprofundar os conhecimentos relacionados às inovações tecnológicas de uma agroindústria específica.

A coleta de dados foi realizada diretamente em uma agroindústria de processamento de feijão, situada no estado do Paraná, fundada em 1996, com cerca de 4.000 metros quadrados de área construída e empregando 65 funcionários diretos e indiretos. Considerando o período da coleta de dados, (11/2015) a empresa processa de 900 a 1.200 toneladas de feijão ao mês, distribuindo seus produtos em comércios de toda região. O processamento da agroindústria objeto de estudo consiste em nove etapas principais, apresentadas na figura 1.

Figura 1: Fluxograma do processamento de feijão

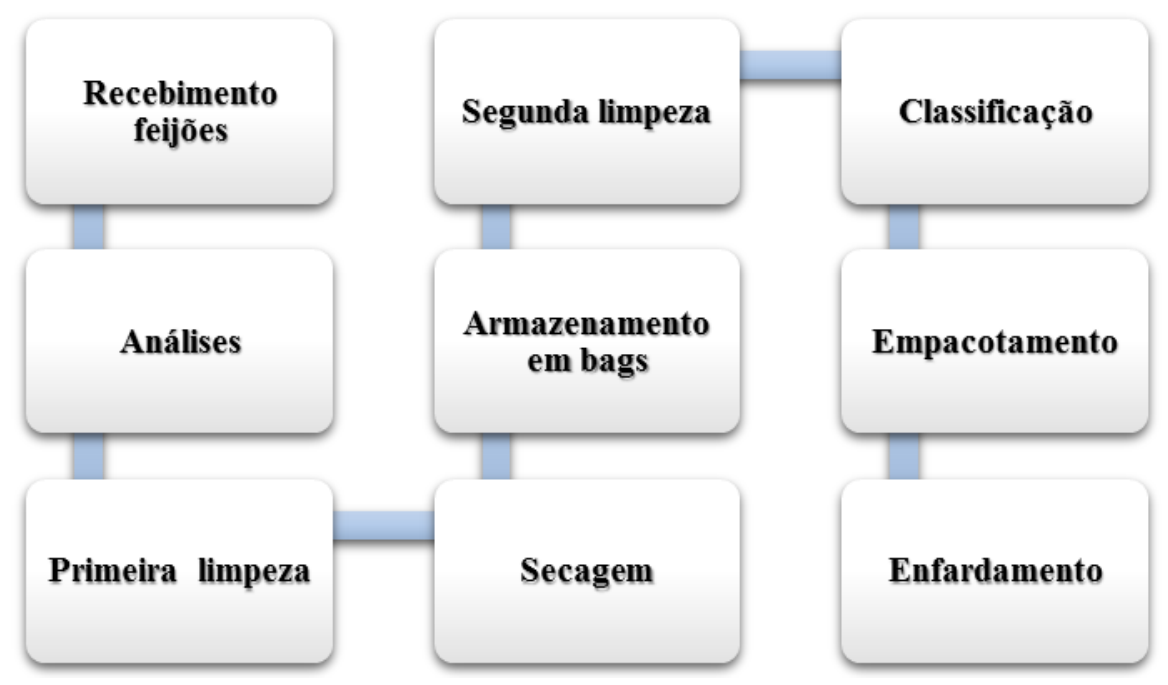

Fonte: Autoria própria (2016)

O fornecimento de matéria-prima é proveniente de 200 produtores rurais da região. Após o recebimento das cargas de feijão, o lote é avaliado quanto à umidade e teor de sujidades. Posteriormente passa pelos processos de limpeza para remoção das sujidades, secagem quando necessária para diminuir a umidade até $14 \%$, seguindo para o armazenamento, que pode durar em média até cinco meses. Durante o beneficiamento, - feijão passa por uma segunda limpeza, objetivando remover as sujidades remanescentes da primeira limpeza e realizar o polimento do grão, seguida da 
classificação (feijão apto para a comercialização ou resíduo), empacotamento (500g) e enfardamento.

Os resíduos provenientes deste beneficiamento são destinados a fabricas de produção de ração animal e distribuídos entre os agricultores da região para utilização no processo de adubação do solo.

\section{Resultados e Discussão}

O beneficiamento dos grãos tem a finalidade de melhorar a qualidade do produto nos aspectos físico, fisiológico e sanitário (ARAUJO et al., 2011). Desta forma, a utilização de equipamentos automatizados podem facilitar este processamento, bem como de garantir maior precisão dos resultados em menor tempo. $\mathrm{Na}$ agroindústria de processamento de feijão foram encontradas inovações tecnológicas de processo nas etapas de limpeza, secagem, classificação e empacotamento, inteiramente automatizados.

Tanto para a primeira, quanto para a segunda limpeza, a indústria usa os equipamentos KW LC160-SP 40TH (figura 2), máquinas no modelo aberto, totalmente metálicas e constituídas de um sistema de recirculação do fluxo de ar de limpeza, em que apenas $15 \%$ do ar utilizado é expelido para o ambiente. Possuem dois tipos de processos de peneiramento: oscilatório circular e retilíneo, ambos com alta eficiência na limpeza (GRUPO KEPLER WEBER, 2016). O processo de limpeza dos grãos antes da armazenagem é uma prática pós-colheita necessária para garantir qualidade e segurança alimentar aos consumidores, visto que os métodos de colheita de feijão não proporcionam um produto final limpo e padronizado em condições de ser comercializado ou consumido (PIRES, 2014).

Figura 2: Máquina de limpeza de grãos modelo aberto KW LC160-SP 40TH

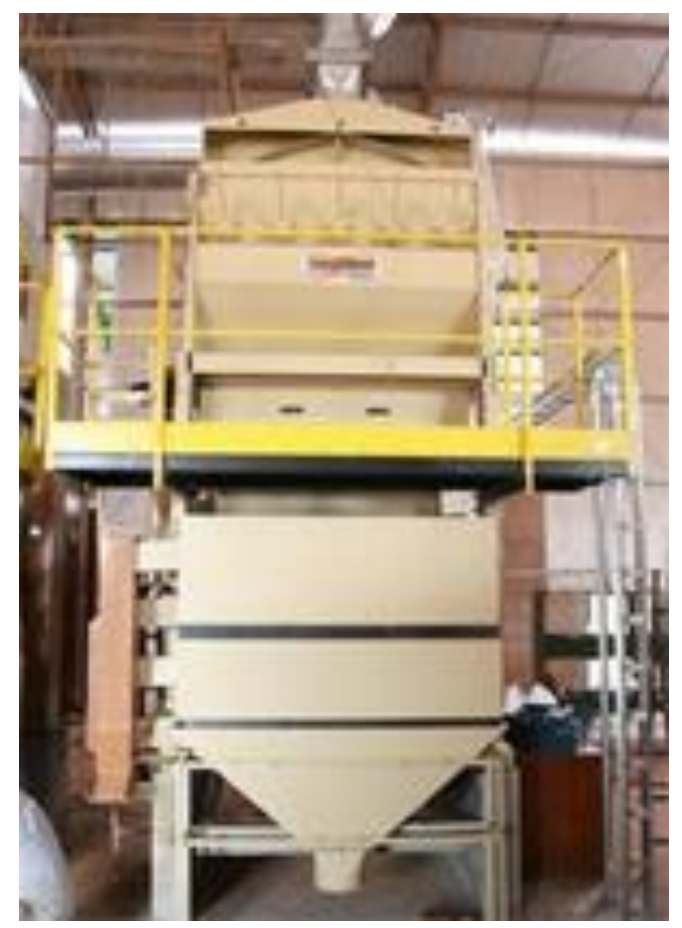

Fonte: Grupo Kepler Weber (2016)

A secagem é realizada por meio do secador rotativo SER-150 (figura 3), com as vantagens de secagem mais rápida e uniforme, economia de combustível e melhor aspecto do produto final, devido a seu sistema de polimento do grão (PINHALENSE, 
2016a). Essa etapa é fundamental para manter a qualidade do produto final, uma vez que o teor de umidade superior a $14 \%$ e atividade água superior a 0,70 podem propiciar aos grãos a proliferação microbiológica, pois a maioria dos bolores, leveduras e bactérias se desenvolvem nessas condições (PIRES, 2014). Segundo a Instrução Normativa $n^{\circ} 12$, de 28 de março de 2008, que estabelece o Regulamento Técnico do Feijão produtos com umidade superior a $14 \%$ podem ser comercializados, desde que não ocasionem riscos à saúde humana.

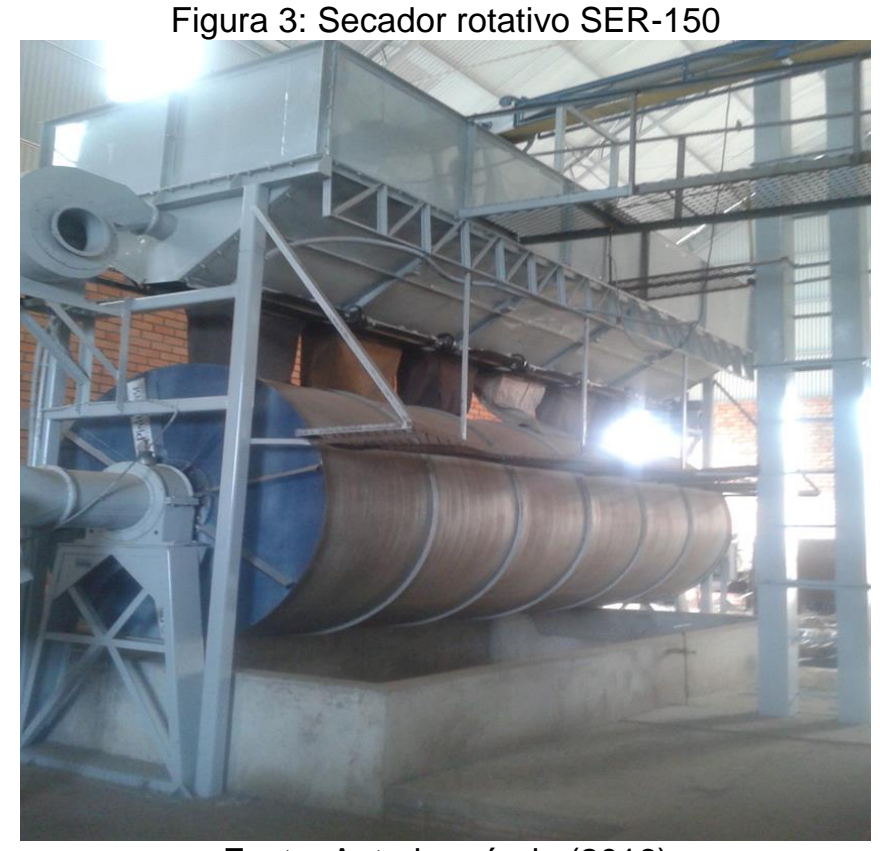

Fonte: Autoria própria (2016)

O processo de classificação durante o beneficiamento do feijão é realizado primeiramente por uma mesa vibratória flutu-ar MVF-2 (figura 4), que classifica os grãos por peso específico, separando os grãos leves, imaturos e atacados por insetos dos grãos inteiros e bem formados (PIRES, 2014). Este equipamento oferece os seguintes benefícios à empresa: baixo consumo de energia, redução da poluição sonora, controle da direção do ar e ajustes simples e diretos (PINHALENSE, 2016b). Após a seleção dos grãos aptos para a comercialização, esses passam pelo processo de separação por cores em um classificador GK-ZC-192 (figura 5).

Figura 4: Mesa vibratória flutu-ar MVF-2

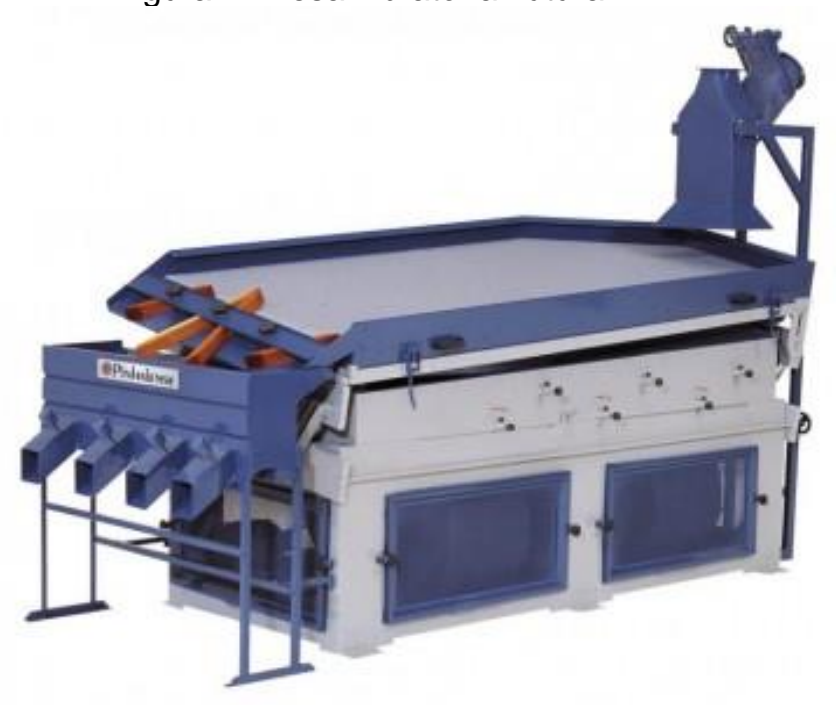

Fonte: Pinhalense (2016) 
Figura 5: Classificador de feijão em cores GK-ZC-192

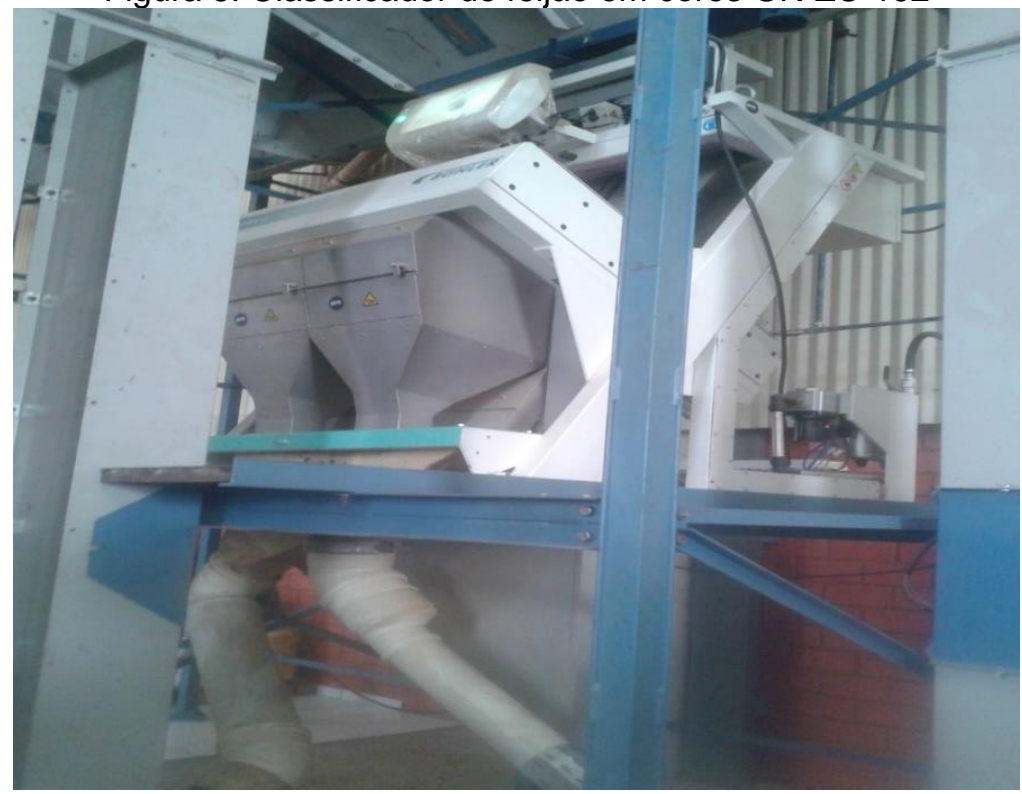

Fonte: Autoria própria (2016)

O empacotamento é realizado por uma empacotadora automática modelo MG-1000 com dosador (figura 6), que possibilita acesso às partes da máquina, facilitando a manutenção, conservação e limpeza do equipamento. Esse equipamento possui sistema de tracionamento do filme por correias de arraste e desbobinador mecânico de filme (INDUMAK, 2016).

Figura 6: Empacotadora automática modelo MG-1000

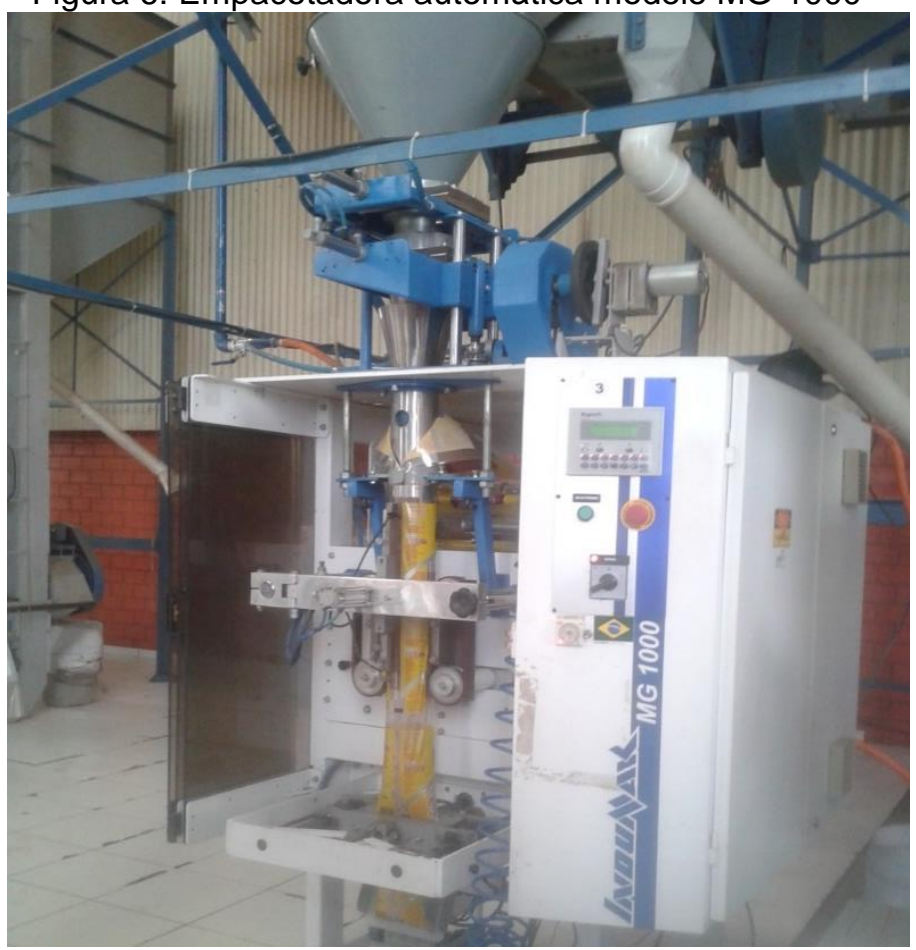

Fonte: Autoria própria (2016)

Tais inovações tecnológicas de processo permitiram à empresa aumentar a produtividade, reduzir os custos operacionais, diminuir o lead time e aumentar a lucratividade. Um estudo semelhante, sobre a importância da inovação tecnológica na indústria de alimentos, indicou que a empresa em estudo apresentou significativas 
atividades inovadoras, cujos resultados provocaram aumento da competitividade em seus mercados de atuação e ganhos internos em termos de eficiência, produtividade e lucratividade (ABREU, 2012).

A agroindústria de processamento de feijão também investiu em inovações de produto, detentora atualmente no mercado do feijão Premium e o feijão vermelho, dois segmentos que vêm ganhando destaque pelo elevado grau de qualidade e inovação oferecidos para o consumidor. Santini, Filho e Bánkuti (2006) relataram em seu estudo que as inovações tecnológicas de produto na cadeia produtiva do café referem-se a cafés especiais (gourmets e orgânicos), novos preparados de café (café com leite, cappuccino), produtos mais padronizados e produtos embalados a vácuo, enquanto que na cadeia produtiva do leite referem-se a novos sabores de iogurtes, derivados em menores embalagens, linha light e produtos adicionados de vitaminas e sais minerais (funcionais). Segundo Beuren, Floriani e Hein (2014), a implementação de inovações de produto é a maneira pela qual as empresas conseguem criar novas expectativas nos consumidores e gerar diferenciações em relação aos demais competidores.

Teixeira et al. (2013), ao estudarem as inovações tecnológicas na caprinocultura, relataram que, dentre as inovações tecnológicas aplicadas, as mais relevantes abrangeram as áreas de reprodução (desde métodos para indução de cio até a prática da transgenia), melhoramento genético (biogenética), sanidade (inovações no diagnóstico e prevenção de doenças de maior impacto na criação), nutrição (inovações nos sistemas de alimentação) e tratamento de dejetos (diminuição de contaminantes no ambiente). Os autores concluíram que a utilização conjunta das inovações tecnológicas nos diferentes segmentos associados à cadeia produtiva caprina pode fazer com que ocorram melhorias nos rebanhos assim como nos produtos gerados.

Nas últimas décadas, a inovação tornou-se um termo inevitável na estratégia de negócios, agendas governamentais e do pensamento acadêmico (ACOSTA; ACOSTA; ESPINOZA, 2016), visto que a introdução de novos ou melhorados métodos de trabalho, de produção e produtos deixaram de serem apenas práticas do setor industrial e passaram a serem ferramentas estratégicas para crescimento no mercado consumidor.

\section{Conclusão}

Abordando este tema, o estudo de caso realizado permitiu perceber o elevado grau de investimento em inovações tecnológicas na agroindústria objeto de estudo. As inovações tecnológicas de processos encontradas referem-se aos processos de limpeza, secagem, classificação e empacotamento, os quais são inteiramente automatizados. As inovações tecnológicas de produtos referem-se ao feijão Premium e o feijão vermelho, atualmente comercializados pela empresa.

Tais inovações tecnológicas permitiram a empresa minimizar os desperdícios, maximizar o lucro, otimizar a produtividade em grande escala, conquistar novos mercados e melhorar a performance organizacional. A partir disso foi possível concluir que a implementação de inovações no setor produtivo é necessária às empresas que anseiam se manter competitivas no mercador consumidor, especialmente para aquelas que estão em um segmento em contínuo desenvolvimento, como o feijão.

\section{Agradecimentos}

Os autores agradecem o suporte financeiro da CAPES. 


\section{Referências}

ABREU, A. A. Importância da inovação tecnológica na indústria de alimentos: um estudo de caso numa empresa de grande porte. In: ENCONTRO NACIONAL DE ENGENHARIA DE PRODUCAO, 22., 2012. Bento Gonçalves. Anais...Rio Grande do Sul: ENEGEP, 2012.

ACOSTA, B.; ACOSTA, M.; ESPINOZA, B. Understanding innovation based on company optics: interpretation mistakes on the types of innovation developed. Revista de Administração e Inovação, São Paulo, v. 13, n.4, p. 295-304, oct./dec. 2016.

ARAUJO, R. F. et al. Fluxograma de beneficiamento para sementes de feijão-mungoverde (Vigna radiata L.) Revista Brasileira de Sementes, Londrina, v. 33, n. 3, p. 387394, dez. 2011.

ARUNDEL, A. et al. The future of innovation measurement in europe: concepts, problems and practical directions. [S.I]: STEP Group/IDEA, 1998.

BEUREN, I. M.; FLORIANI, R.; HEIN, N. Indicadores de inovação nas empresas de construção civil de Santa Catarina que aderiram ao programa brasileiro de qualidade e produtividade no habitat (PBQP-H). Perpectivas em Gestão \& Conhecimento, João Pessoa, v. 4, n. 1, p. 161-178, jan./jun. 2014.

CÁRCEL, J. A. et al. Food process innovation through new technologies: use of ultrasound. Journal of Food Engineering, v. 110, n. 2, p. 200 - 207, may. 2012.

CARVALHO, A. V. et al. Processing and characterization of extruded breakfast meal formulated with broken rice and bean flour. Ciência e Tecnologia de Alimentos, Campinas, v. 32, n. 3, p. 515-524, jul. 2012.

DIACONU, M. Technological innovation: concept, process, typology and implications in the economy. Theoretical and Applied Economics, Romênia, v. 18, n. 10, p. 127-144, 2011.

FACCION, C. E. Qualidade de semente de feijão durante o beneficiamento e armazenamento. 2011. 50 f. Dissertação (Mestrado em Agronomia/Fitotecnia) Programa de Pós-Graduação em Agronomia/Fitotecnica, Universidade Federal de Lavras, Lavras.

GIL, A. C. Métodos e técnicas de pesquisa social. 6. ed. São Paulo: Atlas, 2008.

GRUPO KEPLER WEBER. Máquina de limpeza e pré-limpeza de grãos modelo aberto. Disponível em: < http://br.viarural.com/agricultura/silos/kepler-weber/maquinalimpeza-aberto.htm > Acesso em 19 de set. 2016.

INDUMAK. Empacotadora MG 1000. Disponível em: < http://www.indumak.com.br/produtos/12/43/empacotamento-empacotadoras-mg-1000-emg-8000 >. Acesso em: 20 de set. 2016.

INSTITUTO BRASILEIRO DE GEOGRAFIA E ESTATÍSTICA - IBGE. Pesquisa de orçamentos familiares 2008-2009: análise do consumo alimentar pessoal no Brasil. Rio de Janeiro: IBGE, 2011. 150 p. 
KHANALA, R. et al. Genotypic Association of Parameters Commonly Used to Predict Canning Quality of Dry Bean. Crop Science Society of America, v. 54, n. 6, p. 25642573, nov. 2014.

MINISTÉRIO DA AGRICULTURA, PECUÁRIA E ABASTECIMENTO - MAPA. Feijão. Disponível em: < http://www.agricultura.gov.br/vegetal/culturas/feijao>. Acesso em 14 de ago. 2016.

MINISTÉRIO DA AGRICULTURA, PECUÁRIA E ABASTECIMENTO - MAPA. Instrução Normativa no 12, de 28 de março de 2008. Brasília, 2008.

OECD - ORGANIZAÇÃO PARA COOPERAÇÃO ECONÔMICA E DESENVOLVIMENTO. Manual de Oslo: proposta de diretrizes para coleta e interpretação de dados sobre inovação tecnológica. 1997.

OLIVEIRA, C. A. Inovação da tecnologia, do produto e do processo. Belo Horizonte: Editora de Desenvolvimento Gerencial, 2003.

OLIVEIRA, S. R. M.; ALVES, J. L. Metodologia para avaliar a capacidade de inovação tecnológica na performance de empresas high tech. Revista Gestão Industrial, Ponta Grossa, v. 9, n. 4. p. 830-848, out./dez. 2013.

PATEL, P.; PAVITT, K. Patterns of technological activity: their measurement and interpretation. In: STONEMAN, P. (Org.). Handbook of the economics of innovation and technological change. Oxford: Basil Blackwell Ltd, 1995.

PINHALENSE. Secador rotativo SER-150. Disponível em: $<h t t p: / / w w w . p i n h a l e n s e . c o m . b r / e q u i p a m e n t o . p h p ? i d \_m a q u i n a=379>$. Acesso em: 19 de set. 2016a.

PINHALENSE. Mesa vibratória flutu-ar MVF-2. Disponível em: $<h t t p: / / w w w . p i n h a l e n s e . c o m . b r / e q u i p a m e n t o . p h p ? i d \_m a q u i n a=364>$. Acesso em: 19 de set. 2016b.

PIRES, P. F. F. Qualidade do feijão (Phaseolus Vulgaris l.), classe cores, proveniente da agricultura familiar e do mercado varejista destinado à alimentação escolar. 2014. 108 f. Dissertação (Mestrado em Segurança Alimentar e Nutricional) - Programa de Pós-Graduação em Alimentos e Nutrição, Universidade Federal do Paraná, Curitiba.

SANTINI, G. A.; FILHO, H. M. S.; BÁNKUTI, S. M. S. Inovações tecnológicas em cadeias agroindustriais: alguns casos do segmento de processamento de carnes, leite e café no Brasil. GEPROS, Bauru, v.1, n. 3, p.9-21, ago./jul. 2006.

SNYDER, $\mathrm{H}$. et al. Identifying categories of service innovation: A review and synthesis of the literature. Journal of Business Research, v. 69, n. 7, p. 2401-2408, jul. 2016.

TEIXEIRA, I. A. M. et al. Inovações tecnológicas na caprinocultura. Revista Brasileira de Saúde e Produção Animal, Salvador, v. 14, n.1, p.104-120, jan./mar. 2013. 